\title{
Metais nos Sedimentos em Reservatórios: há Toxicidade Potencial?
}

\section{Metals in the Sediments of Reservoirs: Is There Potential Toxicity?}

\author{
Karen de Souza Ferreira ${ }^{1}$ \\ Bárbara Rani-Borges ${ }^{2}$ \\ Gustavo Laranjeira Melo Santos ${ }^{3}$ \\ Sheila Cardoso-Silva 4 \\ Lilian Rose Marques de Sá ${ }^{5}$ \\ Marcelo Pompêo ${ }^{6}$
}

\begin{abstract}
Palavras-chave:
Metais tóxicos

Guarapiranga

Billings

Rio Grande

Critérios de qualidade

Resumo

Os metais são reconhecidos pelo caráter potencialmente tóxico às comunidades além de bioacumulativos em alguns organismos. No presente trabalho, avaliou-se as concentrações dos metais cádmio, cromo, cobre, chumbo, mercúrio e zinco em sedimentos de três pontos de amostragem nos reservatórios Billings, Guarapiranga e Rio Grande (São Paulo, Brasil) entre os anos 2008 e 2017. Os dados foram comparados à valores de referência regionais, à legislação local e a valores guia de qualidade de sedimento: nível de efeito limiar (TEL), nível de efeito provável (PEL) e Critérios de Avaliação dos Sedimentos (CQS). Amostras de sedimento do reservatório Billings apresentaram os maiores números de metais acima dos valores de referência e acima de PEL. Observou-se elevadas concentrações de cobre nos reservatórios Guarapiranga e Rio Grande, provavelmente em decorrência do sistema de gestão aplicado no local à base de Sulfato de Cobre Pentahidratado. Os dados analisados aqui, indicam que a falta de índices regionalizados, com valores de background e critérios de toxicidade para o sedimento, específicos para esses reservatórios é uma grande deficiência para análises mais acuradas e preditivas. Ainda assim, fica demonstrado que os níveis atuais de contaminação dos sedimentos dos reservatórios estão reduzindo a qualidade da água e pressionando negativamente a biota local e alterando e comprometendo o ecossistema aquático.
\end{abstract}

\section{Keywords: \\ Toxic metal \\ Guarapiranga \\ Billings \\ Rio Grande \\ Guidelines}

\begin{abstract}
Metals are recognized for their potentially toxic character to communities, in addition to being bioaccumulative in some organisms. In this work, the concentrations of cadmium, chromium, copper, lead, mercury, and zinc in sediments from three sampling points in the Billings, Guarapiranga, and Rio Grande reservoirs (São Paulo, Brazil) between the years 2008 and 2017 were evaluated. Data were compared to regional reference values, to local legislation, and to sediment quality values: threshold effect level (TEL), probable effect level (PEL), and Sediment Assessment Criteria (CQS). Billings reservoir sediment samples had the highest number of metals with contents above the reference values and above the PEL. There were high copper concentrations in the Guarapiranga and the Rio Grande reservoirs probably due to the management system currently in use, which is based on Copper Sulphate Pentahydrate. The data analyzed here indicate that the lack of regionalized indexes with background values and toxicity criteria for sediment, specific for each of these reservoirs, is a great problem for more accurate and predictive analysis. This study shows that the current contamination levels of the reservoir sediments are reducing the water quality, negatively pressuring the local biota and altering and compromising the aquatic ecosystem.
\end{abstract}

${ }^{1}$ Universidade Estadual Paulista - UNESP, Sorocaba, SP, Brasil. kferreira@unesp.br

${ }^{2}$ Universidade Estadual Paulista - UNESP, Sorocaba, SP, Brasil. barbara.rani-borges@unesp.br

${ }^{3}$ Universidade Estadual Paulista - UNESP, Sorocaba, SP, Brasil. gustavo.laranjeira@unesp.br

${ }^{4}$ Universidade Federal do Acre (UFAC), Rio Branco, AC, Brasil. she.cardosos@gmail.com

${ }^{5}$ Universidade de São Paulo - USP, São Paulo, SP, Brasil. liliansa@usp.br

${ }^{6}$ Universidade de São Paulo - USP, São Paulo, SP, Brasil. mpompeo@ib.usp.br 


\section{INTRODUÇÃO}

Os ambientes aquáticos têm sido contaminados por substâncias de origem antrópica, destacando-se dentre elas os efluentes industriais e os domésticos. Esses contaminantes podem alcançar diversos compartimentos terrestres e aquáticos, podendo causar efeitos tóxicos na biota (PEREIRA \& EBECKEN, 2009; HUERTA et al. 2013; LÓPEZDOVAL et al., 2017). Entre os poluentes, os metais são os de maior preocupação, pois podem se acumular na cadeia alimentar, formam compostos complexos nocivos que afetam criticamente diferentes funções biológicas e, quando dispostos em reservatórios de abastecimento de água e geração de energia, podem ser extremamente prejudiciais (PAUL et al., 2016; PROSHAD et al., 2018). Tais eventos ocorrem, pois os metais não podem ser degradados por processos naturais e persistem nos sedimentos, de onde são liberados gradualmente aos corpos de água (PAUL; SINHA, 2015). Por esta razão, a contaminação dos sedimentos é considerada por muitas agências reguladoras como um dos principais riscos para o ambiente aquático, uma vez que, muitos organismos passam a maior parte de seu ciclo de vida vivendo em sedimentos (SADIQ; ALAM, 1992).

As propriedades físico-químicas e mineralógicas do sedimento de fundo em ecossistemas aquáticos influenciam o comportamento geoquímico e a biodisponibilidade de metais para a biota aquática. Neste sentido, alterações nas condições desses ambientes exercem papel crucial nos processos de especiação geoquímica, biodisponibilidade e toxicidade de metais (DIAGBOYA et al., 2015; LU et al., 2016). Os contaminantes adsorvidos ao sedimento representam uma fonte de contaminação constante, pelo fato de o sedimento ser um reservatório natural para a deposição destes metais, também sendo um ambiente transformador, já que interage com a biota e a matéria orgânica, e interfere nos teores de oxigênio, fatores esses que podem modificar a toxicidade dos metais ali presentes (SIQUEIRA; APRILLE, 2012; VOIGT et al., 2016).

Metais são compostos químicos potencialmente tóxicos, com propriedades cancerígenas, mutagênicas, teratogênicas e alergênicas, sendo considerados prejudiciais à biota, por isso o seu estudo torna-se tão relevante e o controle desses elementos faz-se imprescindível para minimização e controle dos riscos (MWINYIHIJA, 2011; GODECKE et al.,
2012; BHUSARI, 2016). Dentre os metais mais frequentemente encontrados em ambientes aquáticos e que se destacam pelos níveis tóxicos estão o mercúrio, cobre, cádmio, zinco, cromo e chumbo (LUOMA; RAINBOW, 2008). As ações antrópicas contribuem diretamente para o aumento dos níveis desses metais nos sedimentos (CETESB, 2016), sendo as empresas gestoras responsáveis por analisar as condições e estabelecer critérios e métodos para administrar a problemática.

Os reservatórios Guarapiranga, Billings e Rio Grande abastecem juntos cerca de $30 \%$ do território da Grande São Paulo (SÃO PAULO, 2008), uma região de alta complexidade onde a gestão pública possui grandes desafios, incluindo a problemática da disposição de águas residuais domésticas e industriais e de resíduos sólidos. Resultados de estudos anteriores, indicam que os três reservatórios já apresentavam vulnerabilidade quanto aos níveis de toxicidade do sedimento, principalmente, devido à progressiva deterioração de sua qualidade d'água associada à expansão urbana próxima aos reservatórios (MARIANI; POMPÊO, 2008; POMPÊO et al, 2013; CARDOSO-SILVA et al, 2014). Portanto, o monitoramento contínuo desses mananciais e a avaliação da presença de metais no sedimento é indispensável para um sistema de controle e gerenciamento otimizado dos reservatórios.

Tendo em vista contribuir com os sistemas de gestão públicos, o objetivo deste estudo foi avaliar e comparar as concentrações dos metais cádmio $(\mathrm{Cd})$, cromo $(\mathrm{Cr})$, cobre $(\mathrm{Cu})$, chumbo $(\mathrm{Pb})$, mercúrio $(\mathrm{Hg})$ e zinco $(\mathrm{Zn})$ presentes nos sedimentos de reservatórios paulistas no período de 2008 a 2017. Os resultados foram submetidos a diferentes critérios de avaliação da qualidade de sedimentos, a fim de se observar o potencial de toxicidade desses metais.

\section{MATERIAL E MÉTODOS}

\section{Área de estudo}

A Região Metropolitana de São Paulo (RMSP) é o maior e mais populoso aglomerado urbano do Brasil e um dos cinco maiores do mundo, abrangendo 39 municípios e concentrando mais de 21.4 milhões de habitantes (SÃO PAULO, 2021). A área deste estudo é formada por um complexo hídrico que compreende 2 reservatórios de águas interligados pelo braço de Taquacetuba, ambos com grande população em seus entornos e responsáveis pelo abastecimento de cerca de 6.4 milhões habitantes da RMSP 
(PIRES et al., 2015; POMPÊO; MOSCHINICARLOS, 2012).

O reservatório Guarapiranga é abastecido por uma bacia que recebe o mesmo nome, pelos rios Capivari, Monos e o riacho Taquacetuba (este último fazendo a ligação com o Reservatório Billings). Sua extensão é de aproximadamente $630 \quad \mathrm{~km}^{2}$, abrangendo diferentes municípios da RMSP como EmbuGuaçu, Itapecerica da Serra, São Lourenço da Serra, Juquitiba, Embu e São Paulo. Estima-se que quase 800.000 habitantes moram em suas margens e $60 \%$ deles são residentes na cidade de São Paulo (SABESP, 2008).
O complexo Billings faz divisa com 5 municípios da RMSP e a região sul da cidade de São Paulo, e ainda fornece água por bombeamento à usina hidrelétrica de Henry Borden em Cubatão, para aproveitar o potencial da descida da Serra do Mar (ESCAMES, 2011).

Neste estudo, optou-se por levantar dados de 3 estações de coletas da rede de monitoramento da Companhia Ambiental do Estado de São Paulo - CETESB. Um ponto está localizado no Reservatório Guarapiranga (GUAR 0900)

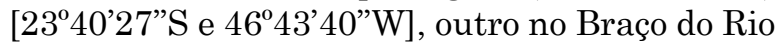
Grande (RGDE 02900) [2346.07"S e $\left.46^{\circ} 32^{\prime} 00^{\prime \prime} \mathrm{W}\right]$ e um terceiro no Reservatório Billings (BILL 02100) [23⒋'57”S 46 38'52”W] (Figura 1).

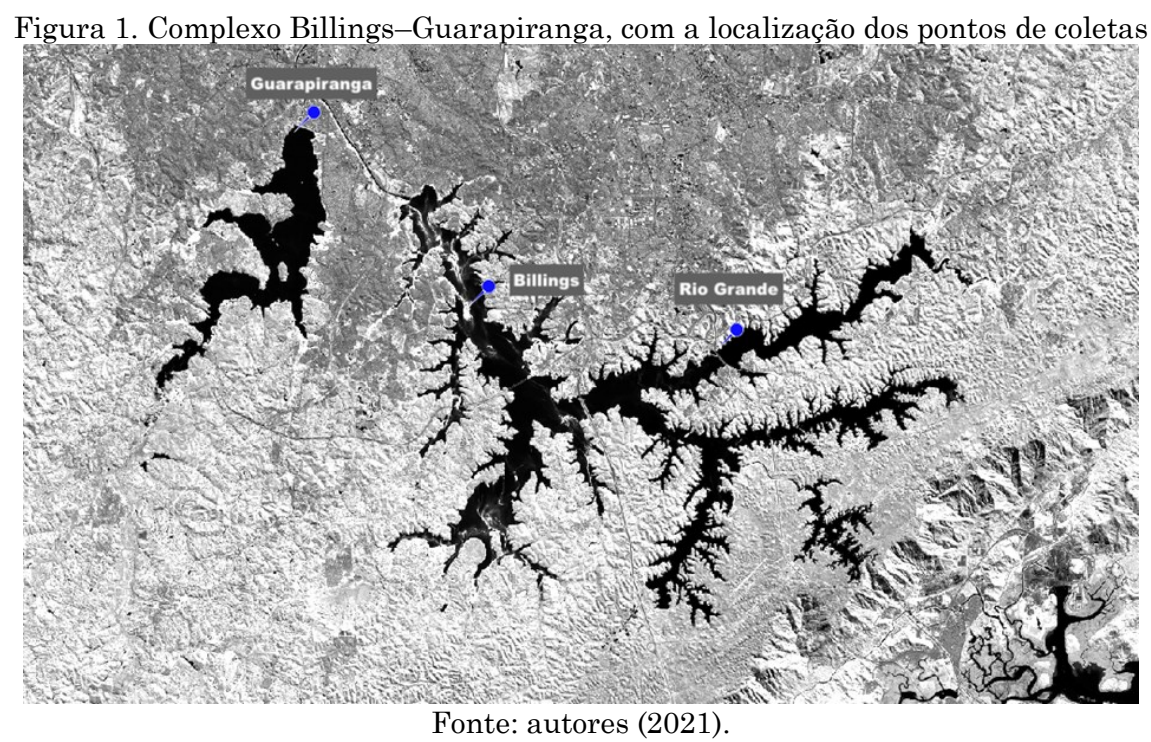

\section{Amostragem e análise laboratorial}

Neste trabalho foram utilizados os dados levantados nas campanhas para a confecção dos relatórios de qualidade de águas superficiais da CETESB, no período de estiagem, outono/inverno (entre abril a setembro) de 2008 a 2017, sendo 4 coletas nos Reservatórios Billings e Guarapiranga e 6 coletas no Braço do Rio Grande (CETESB, 2018). Foram selecionados e compilados os metais de interesse, seja pelo risco toxicológico ou pela contribuição antrópica.

A amostragem foi realizada em triplicata por ponto amostral com distância de $250 \mathrm{~m}$ entre cada lançamento, as amostras de sedimentos superficiais (6 $\mathrm{cm}$ de profundidade) foram coletadas com o auxílio de amostrador de fundo tipo "Van Veen", de 0,020 $\mathrm{m}^{2}$ de embocadura. Em seguida foram retiradas do amostrador com auxílio de pás plásticas e acondicionadas em potes plásticos e transportadas até o laboratório sob refrigeração, e mantidas a $4 \pm 2^{\circ} \mathrm{C}$ até o momento da secagem (ANA, 2011). As amostras de sedimento foram previamente secas a $103^{\circ}$ $105^{\circ} \mathrm{C}$ em estufa, para o mercúrio este processo foi realizado em temperatura abaixo de $60^{\circ} \mathrm{C}$, para que não ocorresse perdas por volatilização, e após este processo, as amostras trituradas e passadas em peneira de 2 milímetros, para homogeneizar, antes da determinação dos metais com espectrometria de emissão óptica com plasma (ICP-OES) seguindo o protocolo 3051A da Agência Ambiental Estadunidense (United States Environmental Protection Agency) - USEPA (2007a). Para mercúrio, o método utilizado foi USEPA 7473 (2007b) através da espectrometria de absorção atômica com decomposição térmica (TDA-AAS).

\section{Análise de dados}

Critérios para avaliação da qualidade de sedimentos

Os valores de concentração para metais nas amostras de sedimento foram comparados com os valores orientadores efeito limiar (TEL, 
threshold effect level) e efeito provável (PEL, probable effect level) do Conselho de Ministros do Meio Ambiente Canadense (CCME, 2001); os Critérios de Avaliação dos Sedimentos - CQS (CETESB, 2008); a Resolução do Conselho Nacional de Meio Ambiente (Conama) $n^{\circ} 454 / 2012$, que orienta nacionalmente as concentrações máximas em corpos hídricos de classe 1 e 2 para sedimentos a serem dragados (BRASIL, 2012) e com valores de referência regionais (VRR) para a o Alto Tietê (NASCIMENTO \& MOZETO, 2008). As informações sobre TEL, PEL, CQS, Conama ${ }^{\circ}$ 454/12 e VRR encontram-se apresentadas nas Tabelas 1 e 2 . Os valores do CQS orientadores foram baseados em variáveis toxicológicas que classificam os sedimentos em cinco classes, excelente, boa, regular, ruim e péssima, oriundas de ensaios de toxicidade aguda/sub- letal com o anfípodo Hyalella azteca e ensaio de mutação reversa (conhecido como teste de Ames), que foram formulados da relação de efeitos biológicos e da relação causa e efeito em vários estágios de vida. Levou-se em conta também a biodisponibilidade e toxicidade do metal no sedimento (CETESB, 2004).

Segundo CCME (2001) os valores-guia adotados em TEL, a probabilidade de o efeito adverso aos organismos ocorrer é praticamente descartada enquanto no PEL, este efeito adverso é provavelmente certo, assim, valores intermediários a TEL e PEL ficam em uma "zona de transição", na qual são incertos os efeitos de toxicidade nos organismos. Já para valores abaixo de VRR, unicamente indicam contribuição natural, decorrente do intemperismo que ocorre na bacia.

Tabela 1. Orientação de toxicidade do sedimento relativamente aos valores de efeito provável, efeito limiar, valores de referência regional para a Bacia do Rio Tietê para os metais analisados e valores de referência estabelecidos pelo CONAMA para corpos d'água Classe 1 e 2. TEL: efeito limiar; PEL: efeito provável; VRR: valores de referência regionais; cádmio $(\mathrm{Cd})$; cromo $(\mathrm{Cr})$; cobre $(\mathrm{Cu})$; chumbo $(\mathrm{Pb})$; mercúrio $(\mathrm{Hg})$; e zinco $(\mathrm{Zn})$.

\begin{tabular}{cccccc}
\hline Metal & TEL & PEL & VRR & $\begin{array}{c}\text { CONAMA } \\
\text { Classe 1 }\end{array}$ & $\begin{array}{c}\text { CONAMA } \\
\text { Classe 2 }\end{array}$ \\
\hline $\mathrm{Cd}$ & 0,6 & 3,5 & $0,22 \pm 0,08$ & 0,6 & 3,5 \\
$\mathrm{Cu}$ & 35,7 & 197 & $18 \pm 6$ & 35,7 & 197 \\
$\mathrm{Cr}$ & 37,3 & 90 & $36 \pm 7$ & 37,3 & 90 \\
$\mathrm{Hg}$ & 0,13 & 0,7 & $0,14 \pm 0,05$ & 0,17 & 0,48 \\
$\mathrm{~Pb}$ & 35,5 & 91,3 & $61 \pm 7$ & 35 & 91,3 \\
$\mathrm{Zn}$ & 123 & 315 & $82 \pm 14$ & 123 & 315 \\
\hline \multicolumn{5}{c}{ Fonte: autores (2021). }
\end{tabular}

Tabela 2. Classificação qualitativa dos sedimentos relativamente à presença dos metais analisados, definida de acordo com os valores de efeito provável (PEL), efeito limiar (TEL) e valores de referência regional (VRR) e as respectivas cores de classificação. Cádmio $(\mathrm{Cd})$; cromo $(\mathrm{Cr})$; cobre $(\mathrm{Cu})$; chumbo $(\mathrm{Pb})$; mercúrio $(\mathrm{Hg})$; e zinco $(\mathrm{Zn})$.

\begin{tabular}{cc|cccc}
\hline \multirow{2}{*}{ Metal } & \multicolumn{5}{c}{ Qualidade do sedimento $\left(\mathrm{mg} \mathrm{kg}^{-1}\right)$} \\
& ÓTIMA & BOA & REGULAR & RUIM & PÉSSIMA \\
\cline { 2 - 6 } $\mathrm{Cd}$ & $<0,24$ & $0,24-0,6$ & $0,60-3,50$ & $3,5-35$ & $>35$ \\
$\mathrm{Cu}$ & $<18$ & $18-35,7$ & $35,7-197$ & $197-1970$ & $>1970$ \\
$\mathrm{Cr}$ & $<30$ & $30-37,3$ & $37,3-90$ & $90-900$ & $>900$ \\
$\mathrm{Hg}$ & $<0,13$ & $0,13-0,16$ & $0,16-0,70$ & $0,7-7$ & $>7$ \\
$\mathrm{~Pb}$ & $<35,5$ & $35,5-61$ & $61-91,3$ & $91,3-913$ & $>913$ \\
$\mathrm{Zn}$ & $<82$ & $82-123$ & $123-315$ & $315-3150$ & $>3150$ \\
\hline Classificação & $<$ VRR & $>$ VRR $<$ TEL & $>$ TEL $<$ PEL & PEL - 10x PEL & $>10 \mathrm{x}$ PEL \\
\hline
\end{tabular}

Fonte: autores (2021).

\section{Análise estatistica}

O processamento estatístico foi realizado por análise de componentes principais (ACP). A ACP foi empregada para auxiliar na compreensão da distribuição e geoquímica dos metais ao longo do tempo e espaço. As análises estatísticas foram desenvolvidas com dados logaritmizados através do software PAST 3.0 (HAMMER et al., 2001).

$\mathrm{Na}$ elaboração da APC, o ano de 2016 foi retirado da análise por não ter ocorrido amostragem em 2 dos 3 reservatórios. Esse fato ocorreu devido à transposição de águas do Braço Taquacetuba (Represa Billings) para a
Guarapiranga, para o abastecimento público durante a crise hídrica na região metropolitana de São Paulo (CETESB, 2016). Para os metais que apresentavam anos como não realizado (NR), foi anotado a média entre os 2 anos subsequentes quando disponível.

\section{RESULTADOS}

Os resultados das análises dos metais nos sedimentos de 2008 a 2017 nos reservatórios Billings, Guarapiranga e Rio Grande estão apresentados na Tabela 3. Nessa tabela 
destacam-se as elevadas concentrações de cobre no Guarapiranga e Rio Grande. Também é possível observar que o Rio Grande apresenta as maiores concentrações de cobre determinadas e menor coeficiente de variação, quando comparado com o Guarapiranga. No entanto, o restante do Complexo Billings também se apresenta com preocupantes concentrações para vários outros metais. Além do $\mathrm{Cu}$ observou-se valores de Hg acima de VRR e sugestivo de efeito tóxico provável à biota principalmente no reservatório Rio Grande.

Tabela 3. Concentrações dos metais ( $\mathrm{mg} \mathrm{kg}^{-1}$ ) cádmio $(\mathrm{Cd})$; cromo $(\mathrm{Cr})$; cobre $(\mathrm{Cu})$; chumbo $(\mathrm{Pb})$; mercúrio $(\mathrm{Hg})$; e zinco $(\mathrm{Zn})$ nos sedimentos superficiais dos reservatórios Billings, Guarapiranga e Rio Grande entre 2008 a 2017 . Os valores são coloridos de acordo com a classificação qualitativa dos sedimentos relativamente à presença dos metais analisados, definida de acordo com os valores de efeito provável, efeito limiar e valores de referência regionais (azul: ótima; verde: boa; amarelo: regular; laranja: ruim; vermelho: péssima). NR: Não realizado; DP: Desvio padrão; CV: Coeficiente de variação; estão destacados em negrito os valores máximos e mínimos para cada metal. Os valores apresentados como $<0,1$ e $<0,5$ estão abaixo do limite de deteç̧ão.

\begin{tabular}{|c|c|c|c|c|c|c|}
\hline \multirow{2}{*}{ Ano } & \multicolumn{6}{|c|}{ Billings ( $\mathrm{mg} \mathrm{kg}^{-1}$ ) } \\
\hline & $\mathrm{Cd}$ & $\mathrm{Cu}$ & $\mathrm{Cr}$ & $\mathrm{Hg}$ & $\mathrm{Pb}$ & $\mathrm{Zn}$ \\
\hline 2008 & $\mathrm{NR}$ & 220 & 237 & 0,62 & 112 & 493 \\
\hline 2009 & 3,76 & 191 & 176 & 0,42 & 149 & 470 \\
\hline 2010 & 3,13 & 191 & 178 & 0,68 & 99,5 & 465 \\
\hline 2011 & 3,71 & 234 & 211 & 0,86 & 79,4 & 568 \\
\hline 2012 & 4,05 & 198 & 189 & 0,77 & 129 & 496 \\
\hline 2013 & 1,68 & 98,9 & 216 & 0,88 & 53,2 & 238 \\
\hline 2014 & 3,37 & 215 & 183 & 0,14 & 105 & 486 \\
\hline 2015 & 3,13 & 216 & 169 & 0,51 & 94,4 & 493 \\
\hline 2016 & NR & NR & NR & NR & NR & NR \\
\hline 2017 & 1,5 & 163 & 124 & 0,9 & 75,5 & 378 \\
\hline Média & 2,4 & 173 & 168 & 0,6 & 90 & 409 \\
\hline DP & 1,5 & 72 & 67 & 0,3 & 42 & 169 \\
\hline $\mathrm{CV}$ & 63 & 42 & 40 & 54 & 46 & 41 \\
\hline \multirow{2}{*}{ Ano } & \multicolumn{6}{|c|}{ Guarapiranga $\left(\mathrm{mg} \mathrm{kg}^{-1}\right)$} \\
\hline & $\mathrm{Cd}$ & $\mathrm{Cu}$ & $\mathrm{Cr}$ & $\mathrm{Hg}$ & $\mathrm{Pb}$ & $\mathrm{Zn}$ \\
\hline 2008 & NR & 1.859 & 72,9 & 0,14 & 77,8 & 119 \\
\hline 2009 & 1,11 & 2.525 & 56,7 & 0,16 & NR & 138 \\
\hline 2010 & 4,34 & 2.269 & 59,1 & 0,32 & 38,5 & 130 \\
\hline 2011 & 0,4 & 1.885 & 37,4 & 0,19 & 35,6 & 92 \\
\hline 2012 & 0,3 & 2.257 & 50,1 & 0,32 & 48,7 & 132 \\
\hline 2013 & 0,92 & 3.991 & 64,4 & 0,14 & 62,8 & 160 \\
\hline 2014 & 0,53 & 916 & 61,5 & $<0,1$ & 75,4 & 99 \\
\hline 2015 & 0,81 & 4.295 & 60,3 & 0,31 & 48,3 & 157 \\
\hline 2016 & NR & NR & NR & NR & NR & NR \\
\hline 2017 & 1,01 & 4.326 & 56,8 & 0,18 & 78,8 & 152 \\
\hline Média & 0,94 & 2.432 & 52 & 0,21 & 47 & 118 \\
\hline $\mathrm{DP}$ & 1,26 & 1.430 & 20 & 0,09 & 29 & 47 \\
\hline $\mathrm{CV}$ & 134 & 59 & 39 & 42 & 63 & 40 \\
\hline \multirow{2}{*}{ Ano } & \multicolumn{6}{|c|}{ Rio Grande (mg kg-1) } \\
\hline & $\mathrm{Cd}$ & $\mathrm{Cu}$ & $\mathrm{Cr}$ & $\mathrm{Hg}$ & $\mathrm{Pb}$ & $\mathrm{Zn}$ \\
\hline 2008 & NR & 3.129 & 84,8 & 4,43 & 62,3 & 335 \\
\hline 2009 & 1,29 & 4.596 & 75,1 & 1,77 & NR & NR \\
\hline 2010 & 0,56 & 2.448 & 75,8 & 7,64 & 50,8 & 101 \\
\hline 2011 & 0,88 & 5.639 & 83,1 & 7,21 & 48,3 & 120 \\
\hline 2012 & 0,94 & 4.317 & 72,1 & 7,20 & NR & 117 \\
\hline 2013 & 0,92 & 4.436 & 74,2 & 9,47 & 50 & 122 \\
\hline 2014 & 0,54 & 4.923 & 41,5 & 2,59 & 48,7 & 107 \\
\hline 2015 & 0,42 & 5.824 & 68,4 & 2,52 & 52,5 & 114 \\
\hline 2016 & $<0,5$ & 5.866 & 52,7 & 2,76 & 59,4 & 120 \\
\hline 2017 & 0,46 & 5.796 & 47,1 & 4,71 & 46,7 & 99,7 \\
\hline Média & 0,65 & 4.697 & 67 & 5 & 42 & 124 \\
\hline $\mathrm{DP}$ & 0,36 & 1.180 & 15 & 2,7 & 23 & 83 \\
\hline $\mathrm{CV}$ & 55 & 25 & 22 & 53 & 54 & 67 \\
\hline
\end{tabular}

Fonte: autores (2021). 
Os resultados da ACP (Fig. 2) mostraram que os metais se agruparam de forma distinta em cada reservatório. A componente 1 explicou $83,2 \%$ da variabilidade dos dados e em conjunto com o eixo 2, explicam $100 \%$. A componente 1 foi influenciada principalmente pelos metais $\mathrm{Cd}$ $(1,0), \mathrm{Cr}(0,95), \mathrm{Pb}(0,99)$ e $\mathrm{Zn}(0,96)$ e os pontos relativos aos resultados do reservatório Billings. $\mathrm{O} \quad \mathrm{Cu}(-0,98)$ e $\mathrm{Hg}(-0,63)$, apresentaram posicionamento negativo em relação a componente 1 e positivo em relação a componente 2 , influenciando a distribuição dos pontos relativos aos resultados do reservatório Rio Grande. A componente 2, influenciada pelos teores de $\mathrm{Hg}(0,77)$. No reservatório Guarapiranga, o metal que tem maior influência é o teor de cobre na região da barragem.

Figura 2. Análise de Componentes Principais (ACP) das concentrações dos metais cádmio, cromo, cobre, chumbo, mercúrio e zinco nos sedimentos superficiais dos reservatórios Billings (B), Guarapiranga (G) e Rio Grande (RG) entre

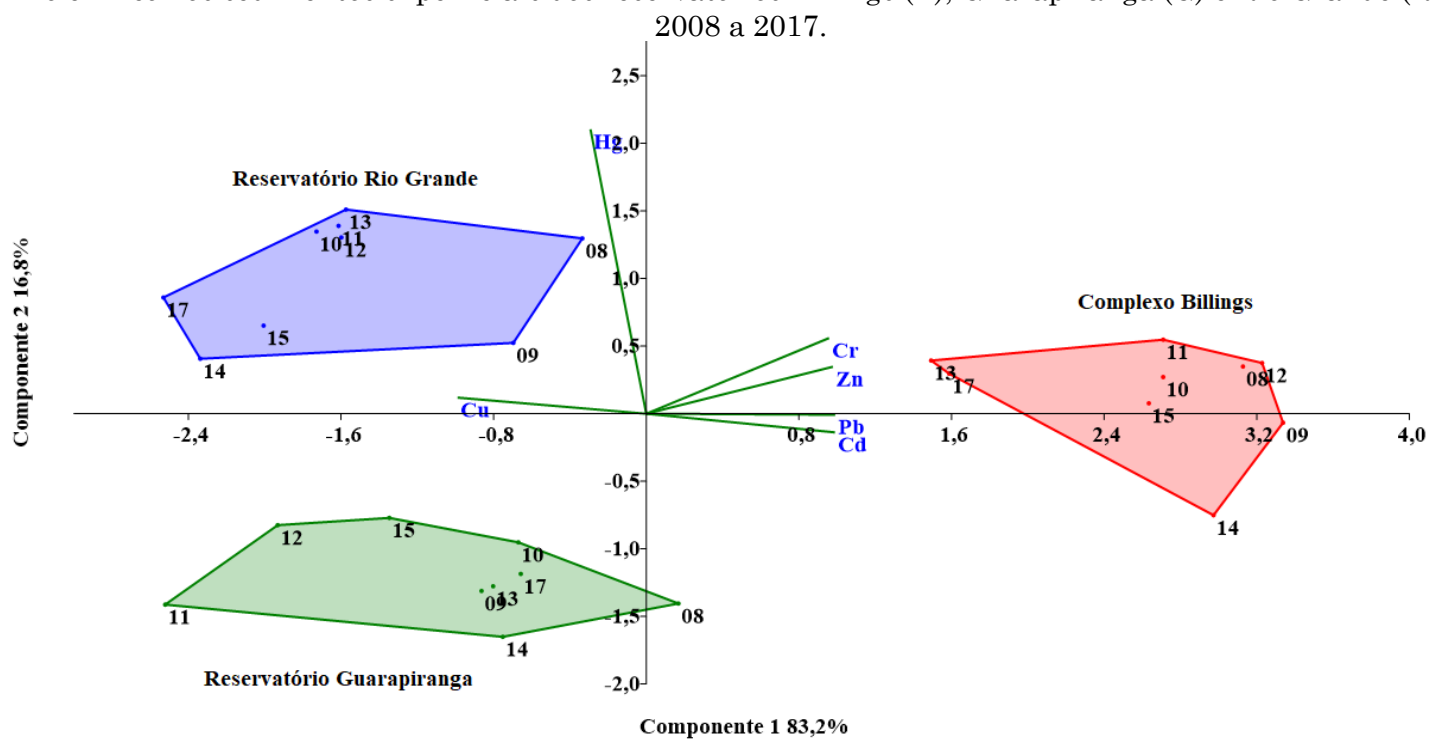

Fonte: autores (2021).

\section{DISCUSSÃO}

Considerando as concentrações médias dos metais em relação aos valores estipulados na resolução $\mathrm{n}^{\circ} 454$ do CONAMA (BRASIL, 2012) para sedimentos em corpos hídricos de classe 1 e 2, os três reservatórios ultrapassaram os valores legais estabelecidos para os metais tóxicos (Tabela 1). O reservatório Guarapiranga esteve em conformidade apenas para o zinco; no reservatório Billings, o cromo é o elemento que mais chama atenção, apresentando praticamente o dobro da concentração permitida para classe 2, enquanto no reservatório Rio Grande, o cobre apresentou concentração até 30 vezes maior do que o estabelecido na resolução e evidencia o grande potencial de contaminação à biota. Estes altos valores também foram observados por Silva et al. (2017), com $24.350 \mathrm{mg}$ $\mathrm{Kg}^{-1}$, e Frascareli et al. (2018), com $2.914 \mathrm{mg} \mathrm{Kg}$ 1 , ambos no reservatório Rio Grande.

A avaliação da qualidade do sedimento pelos valores-guia TEL e PEL indicou que no reservatório Billings o sedimento foi classificado como regular ou ruim para todos os metais, facilmente observado, com base no critério de cores empregadas (Tabela 3). Já os reservatórios Guarapiranga e Rio Grande apresentaram padrão semelhante, com qualidade péssima para cobre e boa para chumbo e zinco, enquanto os demais metais ( $\mathrm{Cd}$ e $\mathrm{Cr}$ ) foram classificados como regular ou ruim.

Chama a atenção os elevados teores de $\mathrm{Cu}$, mas os valores de Hg também são preocupantes, principalmente no reservatório Rio Grande. Além dos valores estarem acima de PEL, exercendo provável efeito tóxico à biota, as condições anaeróbias típicas do hipolímnio do reservatório Rio Grande (MARIANI \& POMPÊO, 2008) podem favorecer a metilação do $\mathrm{Hg}$. Nestas condições o Hg é capaz de atravessar qualquer barreira celular (CRESPO-LÓPEZ et al., 2021) e pode ser biomagnificado, ou seja, suas concentrações tenderiam a aumentar a cada nível trófico. Isto é preocupante, tanto do ponto de vista da saúde ambiental quanto da saúde pública, pois os peixes consumidos na região eventualmente poderão apresentar concentrações acima daquelas recomendadas pela Organização Mundial da Saúde e atingir os consumidores do pescado. O Hg está entre os dez produtos químicos mais tóxicos à saúde pública (WHO, 2017), é um elemento teratogênico e a 
exposição, mesmo a pequenas quantidades, pode levar a consequências deletérias no sistema nervoso central irreversíveis (CRESPO-LÓPES et al., 2021).

O cobre está, geralmente, presente nos sedimentos de reservatórios em grandes concentrações devido às aplicações do algicida sulfato de cobre $\left(\mathrm{CuSO}_{4} .5 \mathrm{H}_{2} 0\right)$, usado no controle da floração fitoplanctônica (PADOVESIFONSECA \& PHILOMENO, 2004). Nos reservatórios paulistas, a aplicação do $\mathrm{CuSO}_{4}$ ocorre desde o final da década de 1970. Nas últimas décadas, o peróxido de hidrogênio, também passou a ser utilizado como alternativa no manejo do crescimento algal, especialmente no de cianobactérias (CETESB 2008; POMPÊO et al. 2013) o que poderia diminuir a quantidade de $\mathrm{CuSO}_{4}$ aplicada. Entretanto, são bastante elevados os teores de $\mathrm{Cu}$ observados nos sedimentos da Guarapiranga e do Rio Grande.

No Rio Grande os valores de concentração de $\mathrm{Cu}$ encontrados foram 136 a 326 vezes superiores aos valores de referência regional (VRR) para o Alto Tietê (utilizando-se os valores máximos e mínimos de concentração e o valor de 18 como valor de VRR) (tabela 2). No reservatório Guarapiranga os valores foram entre 51 a 240 vezes acima do VRR e no reservatório Billings, onde não há aplicação constante do algicida, os valores estiveram 5,5 a 12,2 vezes acima do VRR. De maneira geral são observadas concentrações de $\mathrm{Cu}$ mais altas na região da barragem, local da captação da água bruta, tanto na Guarapiranga (LEAL et al., 2018, POMPÊE et al. 2013) quanto no Rio Grande (MARIANI E POMPÊO, 2008). Estas concentrações mais elevadas na região da barragem é um fator preocupante já que é o local onde ocorre a captação de água para abastecimento público.

Mesmo no reservatório Billings, onde não há aplicações de $\mathrm{CuSO}_{4}$, apenas em 4 anos os valores de $\mathrm{Cu}$ estiveram abaixo de PEL, evidenciando da mesma forma, o grande potencial de contaminação à biota, já que concentrações como as observadas neste trabalho, tem o potencial de oferecer uma grave ameaça às comunidades microbianas do sedimento, de macroinvertebrados bentônicos e de peixes (BEGHELLI et al. 2016; YANG et al. 2018; SUTCLIFFE et al. 2018; XIA et al. 2019).

A presença de metais em concentrações elevadas nos sedimentos pode ser forte indicador da interferência antrópica, possivelmente originado do lançamento de esgotos industriais e da ocupação do solo no entorno da bacia (KORB \& SUERTEGARAY, 2014). Conforme é possível observar na imagem da ACP (Figura 2), existe uma tendência de aumento dos teores de $\mathrm{Cu}$ nos reservatórios Guarapiranga e Rio Grande. Considerando que ambos possuem sistemas de tratamento e gestão semelhantes baseados no emprego de sulfato de cobre, o que pode exercer grande influência na concentração desse metal no sedimento. No complexo Billings a origem das elevadas concentrações de metais provavelmente está associada à esporádica reversão das águas do rio Pinheiros, para regulação da vazão e prevenção de enchentes na capital paulista. Os metais $\mathrm{Cu}, \mathrm{Cd}, \mathrm{Hg}$ e $\mathrm{Zn}$ apresentaram concentrações acima de PEL sugerindo que o sedimento pode causar potencial risco de toxicidade à biota. Valores acima de PEL foram previamente encontrados para o $\mathrm{Cu}$ por Mariani \& Pompêo (2008) (1.644 $\pm 1.067 \mathrm{mg} \mathrm{Kg}^{-1}$ ) e Leal et al. (2018) (1.241 \pm $1.135 \mathrm{mg} \mathrm{Kg}^{-1}$ ), no Rio Grande e Guarapiranga, respectivamente.

Com relação aos metais $\mathrm{Cd}, \mathrm{Pb}$ e $\mathrm{Zn}, \mathrm{o}$ reservatório mais preocupante é o Billings, pois apresenta concentrações em níveis próximos ou acima de TEL; a CSQ qualifica este sedimento como regular ou ruim. Enquanto que para os outros reservatórios, as concentrações destes metais são classificadas como boa, e eventualmente regular em anos pontuais.

Desta maneira, fica evidente que o tipo de gestão e o manejo realizados no reservatório pode contribuir para o incremento da toxicidade do sedimento, como a transposição eventual do Rio Pinheiros, a aplicação de produtos químicos ou mesmo a falta de fiscalização da ocupação irregular do solo no entorno do manancial (FRANKLIN et al., 2016; PADOVESIFONSECA; PHILOMENO, 2004; ALVIM et al., 2015)

Ressalta-se também que, a partir dos resultados da ACP, é possível observar que há um gradiente entre os reservatórios, com o Billings com o menor potencial de toxicidade de seu sedimento, apesar de classificado como ruim, para todos os metais na maioria dos anos estudados, seguido do Guarapiranga com classificação de qualidade ruim para $\mathrm{Cu}$ e regular para os demais metais, na maioria dos anos e posteriormente do Rio Grande, considerado o potencialmente mais tóxico para $\mathrm{Cu}$ (qualidade péssima em todos os anos) e $\mathrm{Hg}$, qualidade péssima e ruim ao longo do período de estudo. Esses resultados estão de acordo com Silva (2013), que desenvolveu estudos comparativos entre os reservatórios do braço Rio Grande, da Billings, Guarapiranga e o Paiva Castro. Os resultados dos testes de toxicidade aguda da água com Daphnia similis sugerem que o braço Rio Grande é potencialmente mais tóxico do que os demais reservatórios estudados. Já os testes de toxicidade aguda do sedimento, 
também com D. similis, foram mais significativos quanto ao potencial tóxico do sedimento do Rio Grande, considerado tóxico em todas as estações e períodos de coleta, seguido do Guarapiranga e por último do Paiva Castro.

Outro aspecto que também deve ser levado em consideração, em decorrência das rotineiras aplicações de $\mathrm{CuSO}_{4}$ e a morte das microalgas, e a posterior lise da parede celular, provocando a liberação de metabólitos na água, como as cianotoxinas (POMPÊO, 2017). As cianotoxinas são metabólitos secundários produzidas pelas cianobactérias e compõem várias classes, com diferentes mecanismos de ação e características próprias e podem causar inúmeros prejuízos à qualidade da água, à saúde humana e dos animais (MANCINI et al., 2010; SÁ et al., 2010; BORTOLI; PINTO, 2015; FONSECA et al., 2015; MACHADO et al., 2016; REGO et al., 2020). Sonobe, Lamparelli e Cunha (2019) indicam que estes episódios de florações tóxicas podem ser mais frequentes se não houver o controle da entrada de matéria orgânica no manancial. Assim, em função dos riscos potenciais que a presença de cianobactérias nos corpos d'água representam, a principal medida preventiva deve ser o controle de seu crescimento desordenado. Este é o motivo da importância de diminuir o enriquecimento dos ecossistemas aquáticos, especialmente no que diz respeito ao nitrogênio e ao fósforo. Esses elementos são os principais responsáveis pelo intenso crescimento do fitoplâncton, particularmente as cianobactérias. Desta maneira, há que ser discutido a adoção de novas formas de gestão e de manejo dos reservatórios paulistas, de modo geral que impeçam o crescimento de cianobactérias potencialmente tóxicas e não empregue rotineiramente algicidas para o controle de seu crescimento.

Com relação a análise temporal, neste trabalho os reservatórios não apresentaram uma mudança brusca nos critérios de qualidade dos sedimentos. Não houve variação suficiente que refletissem em mudanças de risco ecotoxicológico se comparado ao período analisado. No reservatório Billings, a qualidade variou entre regular e ruim, apesar da classificação boa para $\mathrm{Pb}$ e $\mathrm{Hg}$ nos anos de 2013 e 2014, respectivamente. No reservatório Guarapiranga, as concentrações foram enquadradas entre boa e regular, com exceção do Cd em 2010 (ruim) e Hg em 2014 (ótima). Já no reservatório Rio Grande, a variação da qualidade não apresentou a regularidade para a classificação da qualidade da mesma forma como ocorreu para os outros reservatórios, sendo observada melhora nas concentrações de $\mathrm{Pb}$ e $\mathrm{Zn}$, piora para $\mathrm{Hg}$ e regularidade do $\mathrm{Cd}$. Os resultados obtidos, deixam claro que no Rio Grande e no Guarapiranga, devido a aplicação sistemática de algicidas a base de $\mathrm{Cu}$, este elemento teve suas concentrações aumentadas nos reservatórios Rio Grande e Guarapiranga ao longo do tempo, com 2 a 3 vezes os valores da concentração inicial, respectivamente. Segundo Leal et al. (2018), em mais de 40 anos de rotineiras aplicações do sulfato de cobre no Guarapiranga, nesse período, foram estimados em 4.530 toneladas aplicadas no reservatório, unicamente relativo ao elemento $\mathrm{Cu}$.

Por fim, fatores como as altas concentrações de matérias orgânica e $\mathrm{pH}$ podem modificar a toxicidade dos sedimentos, atuando como fases complexadoras de metais e diminuindo a biodisponibilidade desses contaminantes no meio (USEPA, 2005; MARIANI \& POMPÊO, 2011). As porções granulométricas do sedimento também podem favorecer ou não a adsorção de metais, sendo que, quanto menor o tamanho do grão, maiores serão a superfície de contato e, os níveis de complexação (LUOMA \& RAINBOW, 2008).

\section{CONSIDERAÇÕES FINAIS}

A aplicação sistemática de sulfato de cobre, como solução de controle dos organismos fotossintetizantes, que crescem em demasia devido aos altos índices de carga orgânica, nos reservatórios Rio Grande e Guarapiranga, vem provocando o aumento do nível da toxicidade potencial dos sedimentos desses ambientes e colocando este composto químico em evidência. Subsidiar os gestores com dados fidedignos dos níveis de contaminação de sedimentos por metais é essencial e deve ser fundamentado por meio de dados gerados com o monitoramento constante dos reservatórios, que podem ajudar no processo de tomada de decisões para o manejo mais adequado dos recursos hídricos.

Como observado neste trabalho, a comparação das concentrações dos metais do sedimento com valores orientadores de toxicidade e de qualidade sugerem que a biota está em risco, mas é necessária uma visão integradora destes dados, pois o ambiente aquático é dinâmico e muitos fatores podem interagir com os metais e interferir na disponibilização de metais para a coluna d'água e assim, podendo impactar de modo negativo à biota.

A ocupação irregular e o lançamento de efluentes nos reservatórios parecem ser grandes responsáveis pela perda da qualidade dos mananciais estudados, o que intensifica a 
responsabilidade das empresas gestoras. Para garantir uma gestão eficiente, é fundamental o estabelecimento de valores e critérios regionalizados para o sedimento e a construção e implementação de políticas públicas que visem o esgotamento sanitário adequado e a fiscalização da ocupação nos entornos dos mananciais, desta forma, os recursos hídricos provedores da RMSP, estarão salvaguardados da pressão do contínuo crescimento populacional. Portanto, é essencial ter equilíbrio na gestão do recurso hídrico, para que satisfaça mutuamente as necessidades ambientais, da saúde pública e dos diversos usos deste recurso.

Complementarmente, experimentos em laboratório e até mesmo in situ, com testes de toxicidade empregando diversos organismos e o sedimento desses reservatórios, devem ser rotineiramente realizados, visando avaliar os níveis de toxicidade potencial desses sedimentos, não unicamente baseados em guias de qualidade e em concentrações.

\section{AGRADECIMENTOS}

Agradecemos à Fundação de Amparo à Pesquisa do Estado de São Paulo (Processo 2019/10845-4) e ao Conselho Nacional de Desenvolvimento Científico e Tecnológico (processo 303660/20163) pelo financiamento desta pesquisa.

\section{REFERÊNCIAS}

ANA - Agência Nacional de Águas. Guia nacional de coleta e preservação de amostras: água, sedimento, comunidades aquáticas e efluentes líquidos. Carlos Jesus Brandão et al. (Org.). São Paulo: CETESB, Brasília: ANA; 2011. 326p. Disponível em: https://arquivos.ana.gov.br/institucional/sge/CEDO C/Catalogo/2012/GuiaNacionalDeColeta.pdf

Accesso em: Out. 09, 2020

ALVIM, A. T. B.; KATO, V. R. C.; ROSIN, J. R. de G. A urgência das águas: intervenções urbanas em áreas de mananciais. Cadernos Metropoles, v. 17(33), $\quad$ p. $\quad 83-107, \quad 2015$ https://doi.org/10.1590/2236-9996.2015-3304

ARAGÃO, R. B. A.; SEMENSATTO, D.; CALIXTO, L. A.; LABUTO, G. Pharmaceutical market, environmental public policies and water quality: the case of the São Paulo Metropolitan Region, Brazil. Cadernos de Saúde Pública, v. 36, n. 11, e00192319, 2020). https://doi.org/10.1590/0102-311X00192319

AZEVEDO, F.A.; CHASIN, A.A.M. (eds). Metais: Gerenciamento da toxicidade. São Paulo: Editora Atheneu; 2003.
BHUSARI, V. N.; DAHAKE, R.; RAYALU, S.; BANSIWAL, A. Comparative study of removal of hexavalent chromium from water using metal oxide nanoparticles. Advances in Nanoparticles, v. 5(01), p. 67, 2016. http://dx.doi.org/10.4236/anp.2016.51008

BORTOLI, S.; PINTO, E. Cianotoxinas: Características gerais, histórico, legislação e métodos de análises. In: POMPÊO, M. et al. (Org.). Ecologia de reservatórios e interfaces. São Paulo: Instituto de Biociências, v. 1, p. 321-339, 2015. Disponível

em: http://ecologia.ib.usp.br/reservatorios/PDF/Cap._21 _Cianotoxinas.pdf . Acesso em: 12. Nov. 2020.

BRASIL. CONAMA - Conselho Nacional do Meio Ambiente. Resolução $n^{\circ} 454$, de 01 de novembro de 2012. Diário Oficial da União, Brasília, DF, 01 nov. 2012, p. 66. Disponível em: https://www.icmbio.gov.br/cepsul/images/stories/leg islacao/Resolucao/2012/res_conama_454_2012_mat erialserdragadoemaguasjurisdicionaisbrasileiras.p df . Acesso em: 09 Out. 2020

BEGHELLI F. G. S.; POMPÊO M. L. M.; ROSA A. H.; MOSCHINI-CARLOS V. Effects of copper in sediments on benthic macroinvertebrates communities in tropical reservoirs. Limnética. 35(1), p.103-116, 2016. DOI: 10.23818/limn.35.09

CCME - Canadian Council of Ministers of the Environment. Protocol for the derivation of Canadian sediment quality guidelines for the protection of aquatic life. Prepared by the technical secretariat of the CCME task group on water quality guidelines, Ottawa, Canada, p. 35, 2001.

CARDOSO-SILVA, S; NISHIMURA, P Y; PADIAL, P R; MARIANI, C F; MOSCHINI-CARLOS, V; POMPÊO, M L M. Compartmentalization and water quality: Billings reservoir case. Bioikos, 28(1), p. 31-43, 2014. Disponível em: https://periodicos.puc-

campinas.edu.br/seer/index.php/bioikos/article/vie w/2522/1864

CETESB - Companhia Ambiental do Estado de São Paulo. Relatório de Qualidade das águas superficiais no Estado de São Paulo 2003. Anexos. São Paulo. 2004. Disponível em: https://cetesb.sp.gov.br/aguas-interiores/wpcontent/uploads/sites/12/2013/11/anexo2_sediment o.zip. Acesso em: 15 Nov. 2020

CETESB. Relatórios/Publicações. Águas Superficiais. Relatório de Qualidade das Águas Interiores do Estado de São Paulo 2009 a 2019. São Paulo. Base de dados. 2008 a 2018. Disponível em: http://www.cetesb.sp.gov.br/agua/aguassuperficiais 135-publicacoes-/-relatorios. Acesso em: Nov 15, 2020

CRESPO-LOPEZ, M. E.; AUGUSTO-OLIVEIRA, M.; LOPES-ARAÚJO, A.; SANTOS-SACRAMENTO, L.; TAKEDA, P. Y.; MACCHI, B. M.; NASCIMENTO, J. L. M.; MAIA, C. S. F.; LIMA, R. R.; ARRIFANO, G. P. MERCURY: What can we learn from the Amazon? Environment International, 146, e.106223, 2021. https://doi.org/10.1016/j.envint.2020.106223

DIAGBOYA, P. N.; OLU-OWOLABI, B. I.; ADEBOWALE, K. O. Effects of time, soil organic 
matter, and iron oxides on the relative retention and redistribution of lead, cadmium, and copper on soils. Environmental Science Pollution Research. v. 22, p. 10331-10339, 2015. DOI: 10.1007/s11356-0154241-0

FONSECA, J. R.; VIEIRA, P. C. S.; KUJBIDA, P.; COSTA, I. A. S. Cyanobacterial occurrence and detection of microcystins and saxitoxins in reservoirs of the Brazilian semi-arid. Acta Limnologica Brasiliensia, v. 27(1), p. 78-92, 2015. https://doi.org/10.1590/S2179-975X2814

ESCAMES, E. F. USINA PARQUE: Aproveitamento e valorização do patrimônio energético, ambiental e histórico da Usina Hidrelétrica de Henry Borden Dissertação de Mestrado - Santo André, SP. 2011 Universidade Federal do ABC. Disponível em: http://biblioteca.ufabc.edu.br/index.php?codigo_sop hia=15032. .Acesso em: Nov. 11, 2020

FRASCARELI, D.; CARDOSO-SILVA, S.; MIZAEL, J. de O. S. S.; ROSA, A. H.; POMPÊ, M. L. M.; LÓPEZ-DOVAL, J. C.; MOSCHINI-CARLOS, V. Spatial Distribution, Bioavailability, and Toxicity of Metals in Surface Sediments of Tropical Reservoirs, Brazil. Environmental Monitoring and Assessment, v.190(4), 199, 2018. DOI: 10.1007/s10661-018-65158

FRANKLIN, R. L.; FÁVARO. D. I. T.; DAMATTO, S. R. Trace metal and rare earth elements in a sediment profile from the Rio Grande reservoir, São Paulo, Brazil - determination of anthropogenic contamination, dating and sedimentation rates. Journal of Radioanalytical and Nuclear Chemistry, v. 307, p. 99-110, 2016. DOI

https://doi.org/10.1007/s10967-015-4107-4

GODECKE, M. V.; RODRIGUES, M. A. S.; NAIME, R. H. Resíduos de curtumes: estudo das tendências de pesquisa. Revista Eletrônica em Gestão, Educação e Tecnologia Ambiental. v. 7(7), p. 1357 1378 , 2012.

DOI http://dx.doi.org/10.5902/223611705779

HAMMER, O.; HARPER, D. A. T.; RYAN, P. D. Past: Paleontological Statistics Software Package for Education and Data Analysis. 2001. Disponível em: https://palaeo-electronica.org/2001_1/past/past.pdf. Acesso em: Nov 15, 2020

HUERTA, B.; MARTI, E.; GROS, M.; LÓPEZ, P.; POMPÊO, M.; ARMENGOL, J.; BARCELÓ, D. BALCÁZAR, J. L.; RODRÍGUEZ-MOZAZ, S.; MARCÉ, R. Exploring the links between antibiotic occurrence, antibiotic resistance, and bacterial communities in water supply reservoirs. Science of the Total Environment, v. 456-457, p. 161-170, 2013. https://doi.org/10.1016/j.scitotenv.2013.03.071

KORB, C. C. \& ANTUNES-SUERTEGARAY, D. M. Identificação de depósitos tecnogênicos em um reservatório de abastecimento de água da cidade de Pelotas (RS). Quaternary and Environmental Geosciences, [S.l.], v.5(1), 2014 http://dx.doi.org/10.5380/abequa.v5i1.33918

LEAL， P. R.; MOSCHINI-CARLOS, V.; LÓPEZDOVAL, J. C.; CINTRA, J.P.; YAMAMOTO, J. K.; BITENCOURT, M. D.; SANTOS, R. F.; ABREU, G. C.; POMPÊO, M. L. M. Impact of copper sulfate application at an urban Brazilian reservoir: A geostatistical and ecotoxicological approach. Science of the Total Environment, v. 618, p. 621-634, 2018. https://doi.org/10.1016/j.scitotenv.2017.07.095

LÓPEZ-DOVAL, J. C.; MONTAGNER, C. C.; ALBURQUERQUE, A. F.; MOSCHINI-CARLOS, V.; UMBUZEIRO, G.; POMPÊO, M. Nutrients, emerging pollutants and pesticides in a tropical urban reservoir: Spatial distributions and risk assessment. Science of the Total Environment, v. 575, p. 1307-1324, 2017. https://doi.org/10.1016/j.scitotenv.2016.09.210

LU, J.; YUAN, F.; ZHANG, F.; ZHAO, Q. The study on heavy metal distribution in the sediment of middle tidal flat in Yangtze Estuary, China. Environmental Earth Sciences, v. 75(7), p.1-12, 2016. https://doi.org/10.1007/s12665-016-5356-4

LUOMA, S. N. \& RAINBOW, P. S. Metal Contamination in Aquatic Environments: Science and lateral management. Cambridge: Cambridge University. 2008.

p. $573 . \quad$ https://doi.org/10.1111/j.10958649.2009.02440_4.x

MACHADO, L. S.; SANTOS, L. G.; LÓPES-DOVAL, J. C.; POMPÊO, M.; MOSCHINI-CARLOS, V. Fatores ambientais relacionados à ocorrência de cianobactérias potencialmente tóxicas no reservatório de Guarapiranga, SP, Brasil. Revista Ambiente \& Água, v. 11(4), p. 810-818, 2016. https://doi.org/10.4136/ambi-agua.1941

MANCINI, M.; RODRIGUEZ, C.; BAGNIS, G.; LIENDO, A.; PROSPERI, C.; BONANSEA, M.; TUNDISI, J. G. Cianobacterial bloom and animal mass mortality in a reservoir from Central Argentina. Brazilian Journal of Biology, v. 70(3), p. 841-845, 2010. https://doi.org/10.1590/S151969842010000400015

MARIANI, C. \& POMPÊO, M. L. M. Potentially bioavailable metals in sediment from a tropical polymictic environment - Rio Grande Reservoir, Brazil. Soil \& Sediment Contamination, v. 8(5), p. 284-288, 2008. https://doi.org/10.1007/s11368-0080018-0

MARIANI, C. \& POMPÊO, M. L. M. Sedimento: como avaliar sua contaminação por metais. Saneas, 12(40):10-13, 2011.

MWINYIHIJA, M. Essentials of ecotoxicology in the tanning industry. Journal of Environmental Chemistry and Ecotoxicology. v. 3(13), p. 323-331, 2011. https://doi.org/10.5897/JECE11.066.

NASCIMENTO, M. R. L. \& MOZETO, A. Reference value for metals and metalloids concentrations in botton sediments of Tietê river basin, southeast of Brazil. Soil \& Sediment Contamination: an International Journal, v. 17(3), p. 269-278, 2008. https://doi.org/10.1080/15320380802006996

PADOVESI-FONSECA, C. \& PHILOMENO, M. G. Effects of algicide (Copper Sulfate) application on short-term fluctuations of phytoplankton in Lake Paranoá, Central Brazil. Brazilian Journal of Biology, 64(4), p. $\quad 819-826, \quad 2004$. https://doi.org/10.1590/S1519-69842004000500011

PAUL, D. \& SINHA, S. N. Isolation and characterization of a phosphate solubilizing heavy metal tolerant bacterium from River Ganga, West Bengal, India. Songklanakarin Jounal of Science \& Technology, v. 37(6), p. 651-657, 2015. 
https://rdo.psu.ac.th/sjstweb/journal/37-6/37-67.pdf

PAUL, B.; SINGH, K.; JARON, T.; ROY, A.; CHOWDHURY, A. Structural properties and the fluorite-pyrochlore phase transition in $\mathrm{La}_{2} \mathrm{Zr}_{2} \mathrm{O}_{7}$ : the role of oxygen to induce local disordered states. Journal of Alloys and Compounds, v. 686, p. 130-136, https://doi.org/10.1016/j.jallcom.2016.05.347

PEREIRA, G. C.; EBECKEN, N. F. F. Knowledge discovering for coastal waters classification. Expert Systems with Application, 36(4), p. 8604-8609, 2009. https://doi.org/10.1016/j.eswa.2008.10.009

PIRES, D. A.; TUCCI, A. CARVALHO, M. do C.; LAMPARELLI, M. C. Water quality in four reservoirs of the metropolitan region of São Paulo, Brazil. Acta Limnologica Brasiliensia, 27(4), p. 370380, 2015. https://doi.org/10.1590/S2179-975X4914

POMPÊO, M. \& MOSCHINI-CARLOS, V. O Abastecimento de água e o esgotamento sanitário: propostas para minimizar os problemas no Brasil. In ROSA, A. H.; FRACETO, L.F.; CARLOS, V. M. Meio ambiente e sustentabilidade. 1. ed. Porto Alegre-RS: Editora Bookman, 2012, p. 412.

POMPÊO, M.; PADIAL, P. R.; MARIANI, C. F.; CARDOSO-SILVA, S.; MOSCHINI-CARLOS, V.; SILVA, D. C. V. R. DA; PAIVA, T. C. B. DE; BRANDIMARTE, A. L. Biodisponibilidade de metais no sedimento de um reservatório tropical urbano (reservatório Guarapiranga - São Paulo (SP), Brasil): há toxicidade potencial e heterogeneidade espacial? Geochimica Brasiliensis, v. 27(2), p. $104-119, \quad 2013$. http://dx.doi.org/10.21715/gb.v27i2.364

POMPÊO, M. O Controle da Flora e Fauna Aquáticas pela Resolução CONAMA 467: Considerações Sobre a Normativa Brasileira. Revista do Departamento de Geografia, v. 33, p. 24-35, 2017. https://doi.org/10.11606/rdg.v33i0.121065

PROSHAD, R.; KORMOKER, T.; MURSHEED, N.; ISLAM, M.; BHUYAN, I.; ISLAM, S.; MITHU, T. N. Heavy metal toxicity in agricultural soil due to rapid industrialization in Bangladesh: a review. International Journal of Advanced Geosciences, [S.l.], v.6(1), p. 83-88, 2018. http://dx.doi.org/10.14419/ijag.v6i1.9174

REGO, A. H. G.; RANGEL-JUNIOR, A.; COSTA, I. A. S. Phytoplankton scenario and microcystin in water during extreme drought in semiarid tropical water supplies, Northeastern Brazil. Brazilian Journal of Biology, v. $80(1), \quad$ p. $1-11,2020$. https://doi.org/10.1590/1519-6984.182599

SÁ, L. L. C.; VIEIRA, J. M. S.; MENDES, R. A.; PINHEIRO, S. C. C.; VALE, E. R.; ALVES, F. A. S.; JESUS, I. M.; SANTOS, E. C. O.; COSTA, V. B. Ocorrência de uma floração de cianobactérias tóxicas na margem direita do Rio Tapajós, no Município de Santarém (Pará, Brasil). Revista PanAmazônica de Saúde, v. 1(1), p. 159-166, 2010. DOI: 10.5123/S2176-62232010000100022

SABESP - Companhia De Saneamento Básico do Estado de São Paulo. Dossiê - Sistema Guarapiranga. São Paulo: SABESP, p. 16, 2008. Disponível em: http://memoriasabesp.sabesp.com.br/acervos/dossie s/pdf/9_sistema_guarapiranga.pdf. Acesso em: Nov 20,2020

SADIQ, M.; ALAM, I. A. Bioaccumulation of mercury by clams (Meretrix meretrix) collected from the Saudi Coast of the Arabian Gulf. Chemical Speciation \& Bioavailability, v. 4(1), p. 9-17, 1992. https://doi.org/10.1080/09542299.1992.11083173

SÃO PAULO (Estado) Secretaria do Meio Ambiente. Coordenadoria de Educação Ambiental. Caderno Ambiental Guarapiranga Guarapiranga. Secretaria de Estado do Meio Ambiente. Coordenadoria de Educação Ambiental. São Paulo: SMA/CEA, 2008. Disponível

em: http://arquivos.ambiente.sp.gov.br/cea/2015/06/Cad erno-Ambiental-Guarapiranga.pdf. Acesso em: 20 Out. 2020

SÃO PAULO (Estado) Fundação Sistema Estadual de Análise de Dados (SEADE). Sistema Seade de projeções populacionais. Secretaria de Governo do Estado de São Paulo. 2021. Disponível em: http://produtos.seade.gov.br/produtos/projpop/index .php. Acesso em: 23 Jun. 2021.

SILVA, L. S.; FERREIRA, F. J.; FÁVARO, D. I. T. Avaliação da concentração de metais tóxicos em amostras de sedimentos dos reservatórios do complexo Billings (Guarapiranga e Rio Grande). Geochimica Brasiliensis, 31(1), p. 37-56, 2017. https://doi.org/10.21715/GB2358-2812.2017301037

SILVA, D. C. V. R. da. Toxicidade da água $e$ sedimento dos reservatórios Guarapiranga, Billings e Paiva Castro, na Região Metropolitana de São Paulo. São Paulo. 2013. Dissertação (Mestrado) Departamento de Ecologia, Instituto de Biociencias, Universidade de São Paulo. Disponível em: https://www.teses.usp.br/teses/disponiveis/41/4113 4/tde-23102013

091653/publico/Daniel_Silva_CORRIG.pdf. Acesso em: 20 Out. 2020

SIQUEIRA, G. W. \& APRILE, F. M. Distribuição de mercúrio total em sedimentos da plataforma continental Amazônica: Brasil. Acta Amazonica, 42(2), p. 259-268, 2012. https://doi.org/10.1590/S0044-59672012000200012

SONOBE, H. G.; LAMPARELLI, M.C.; CUNHA, D.G.F. Avaliação espacial e temporal de aspectos sanitários de reservatórios com captação de água para abastecimento em SP com ênfase em cianobactérias e cianotoxinas. Engenharia Sanitária e Ambiental, 24(5), p. 909-918, 2019. https://doi.org/10.1590/S1413-41522019193351

SUTCLIFFE, B.; CHARITON, A. A.; HARFORD, A. J.; HOSE, G. C.; GREENFIELD, P.; MIDGLEY, D. J.; PAULSEN, I. T. Diverse fungal lineages in subtropical ponds are altered by sediment-bound copper. Fungal Ecology, 34: p.28-42, 2018. https://doi.org/10.1016/j.funeco.2018.03.003

US EPA - United States Environmental Protection Agency. Procedures for the Derivation of Equilibrium Partitioning Sediment Benchmarks (ESBs) for the Protection of Benthic Organisms: Metal Mixtures. 2005.

US EPA - United States Environmental Protection Agency. Method 3051a - Microwave assisted acid digestion of sediments, sludges, soils, and oils. Revision. 2007a Disponível em: 
https:/www.epa.gov/sites/production/files/2015-

12/documents/3051a.pdf. Acesso em: 2 Nov. 2020

US EPA - SW 846 - Test Methods for Evaluating Solid Waste, Physical/Chemical Methods Environment Protection Agency, USA. 2007b. Disponível em: http://www.epa.gov/waste/hazard/testmethods/sw8 46/online/index.htm. Acesso em: 2 Nov. 2020

VOIGT, C. L.; SILVA, C. P. da; CAMPOS, S. X. de. Avaliação da bioacumulação de metais em Cyprinus carpio pela interação com sedimento e água de reservatório. Química Nova,39(2), p. 180-188, 2016. http://dx.doi.org/10.5935/0100-4042.20160014

WHO - World Health Organization. Mercury and health. 2017.2 Disponível em: https://www.who.int/news-room/factsheets/detail/mercury-and-health. Acesso em: 10 Dez 2020.

XIA W.; CHEN L.; DENG X.; LIANG G.; GIESY J. P.; RAO Q.; WEN Z.; WU Y.; CHEN J.; XIE P. Spatial and interspecies differences in concentrations of eight trace elements in wild freshwater fishes at different trophic levels from middle and eastern China. Science of the Total Environment, 672: p. 883-892,

2019. https://doi.org/10.1016/j.scitotenv.2019.03.13 4
YANG, J.; XIE, Y.; JEPPE, K.; LONG, S.; PETTIGROVE, V.; ZHANG, X. Sensitive Community Responses of Microbiota to Copper in Sediment Toxicity Test. Environmental Toxicology and Chemistry, 37(2), p. 599-608, 2018. https://doi.org/10.1002/etc.3980

\section{CONTRIBUIÇÃO DOS AUTORES}

Karen de Souza Ferreira conceituou, fez a pesquisa de literatura, analisou os dados e escreveu. Bárbara Rani-Borges realizou a pesquisa bibliográfica, analisou os dados e escreveu. Gustavo Laranjeira Melo Santos realizou a pesquisa bibliográfica, analisou os dados e escreveu. Sheila Cardoso-Silva Curadoria redigiu, revisou e editou os dados. Lilian Rose Marques de Sá realizou a curadoria, redigiu, revisou e editou os dados. Marcelo Pompêo administrou os projetos, supervisou, realizou a curadoria de dados, redigiu, revisou e editou. 